\title{
ETA INVARIANTS OF DIRAC OPERATORS ON FOLIATED MANIFOLDS
}

\author{
GORAN PERIĆ
}

\begin{abstract}
We define the eta function of Dirac operators on foliated manifolds. We show that the eta functions are regular at the origin thus defining corresponding eta invariants of foliated manifolds. Under the hypothesis of invertibility of the operator in question, we prove the Atiyah-Singer relative index theorem for Dirac operators on foliated manifolds. Then we discuss the homotopy invariance of the index with respect to secondary data.
\end{abstract}

A foliation of the smooth manifold $M$ is a partition of $M$ into $p$-dimensional submanifolds, called leaves, so that the tangent spaces to the leaves form an integrable bundle. Locally, a foliation is a fibration. However, a leaf can pass infinitely often through the given coordinate patch so that globally the situation is much more complicated.

Foliations carry interesting differential operators such as the Laplace-Beltrami operator, Dirac and signature operators, etc. In this setting one would expect to have a sort of index theorem, ind $(D)=$ average index over the leaves, analogous to the Atiyah-Singer index theorem for fibrations. There are two essential difficulties.

First, leaves of a compact foliation may be noncompact so that the operator under consideration can have an infinite-dimensional kernel or cokernel, and thus the index makes no sense. Second, the space of leaves is not in general a standard Borel measure space so that there is no way of averaging over it.

A. Connes has realized that the space of leaves can be replaced by a "noncommutative space," that is a $C^{*}$ algebra $C_{r}^{*}(G)$ of the holonomy groupoid $G$. In the case of fibrations $F \rightarrow M \rightarrow B$ this algebra is isomorphic to $C(B) \otimes \mathscr{K}$, where $\mathscr{K}$ is the algebra of compact operators on $L^{2}(F)$. That suggested $K_{0}\left(C_{r}^{*}(G)\right)$ as a home for the index. Imposing the condition of the invariant transverse measure $\nu$ on the foliation gives rise to a trace $\Phi_{\nu}$ on $C_{r}^{*}(G)$ and thus a real number $\operatorname{ind}_{\nu}(D)=\Phi_{\nu}(\operatorname{ind}(D)) \in \mathbf{R}$. This is an analytic index.

Connes Index Theorem. $\operatorname{ind}_{\nu}(D)=\left\langle\operatorname{ch}(D) \operatorname{Todd}(M),\left[C_{\nu}\right]\right\rangle$.

Here $C_{\nu}$ stands for the Ruelle-Sullivan current associated to the invariant transverse measure $\nu$.

Received by the editors November 29, 1989 and, in revised form, June 11, 1990 and September 4, 1990.

1980 Mathematics Subject Classification (1985 Revision). Primary 58G10, 53C12; Secondary $47 \mathrm{C} 15,58 \mathrm{~A} 10,58 \mathrm{~A} 25$. 
This article is divided into three parts. In the first part we consider an analogue of the Atiyah-Patodi-Singer eta function of the Dirac-type operator on the foliated manifold $M$. In the second part we derive the generalization of the Atiyah-Patodi-Singer relative index theorem for Dirac operators. In the third part we conjecture the index formula for foliated cylinder and discuss the homotopy invariance of $\operatorname{ind}_{\nu}^{1}$ with respect to secondary data. Our basic results are the following:

Theorem A. Let $\mathscr{D}: \Gamma_{\tau}^{\infty}(M, S) \rightarrow \Gamma_{\tau}^{\infty}(M, S)$ be a tangential Dirac-type operator on the foliated manifold $M$. Then its eta function,

$$
\eta(\mathscr{D}, s)=\Phi_{\nu}\left(\mathscr{D} \exp \left(-t \mathscr{D}^{2}\right)\right)
$$

is a well-defined and meromorphic function for $\operatorname{Re}(s) \leq 0$ with eventually simple poles at $(\operatorname{dim} \mathscr{F}-k) / 2(k=0,1,2, \ldots) . \eta(\mathscr{D}, s)$ is regular at $s=0 .{ }^{1}$

Theorem B. Let $(M, \mathscr{F})$ be a foliated spin manifold of odd dimension $p$ endowed with the holonomy invariant measure $\nu$. Let $S \rightarrow M$ be a corresponding tangential spin bundle and $E \rightarrow M$ a tangentially smooth bundle. Let $\mathscr{D}_{u}: \Gamma_{\tau}^{\infty}(M, S \otimes E) \rightarrow \Gamma_{\tau}^{\infty}(M, S \otimes E), 0 \leq u \leq 1$, be a family of $(\nu$-a.e. $)$ invertible Dirac operators associated with the family $\nabla_{u}^{E}$ of connections on the bundle $E$ and the spinor connection $\nabla^{S}$. Then

$$
\left.\frac{d \eta\left(\mathscr{D}_{u}, s\right)}{d u}\right|_{s=0}=\int_{M} a_{p}\left(x, \dot{\mathscr{D}}_{u}, \mathscr{D}_{u}^{2}\right) d \nu
$$

where the integrand is a certain tangential form which as a class in $\bar{H}_{\tau}^{p}(M, \mathscr{F})$ is given by

$$
a_{p}\left(x, \dot{\mathscr{D}}_{u}, \mathscr{D}_{u}^{2}\right)=\sum_{4 i+2 j=p-1}\left(\frac{\sqrt{-1}}{2 \pi}\right)^{j} \frac{1}{(j-1) !} \widehat{A}_{\tau, i} \cap \operatorname{tr}\left(\psi \cap \Omega_{E}^{j-1}\right) .
$$

$\Omega_{E}=\Omega_{E}(u)$ is the curvature of $\nabla_{u}^{E}$ and $\psi$ is a 1-form $\nabla_{1}^{E}-\nabla_{0}^{E}$.

Theorem $\mathrm{C}$. On the cosphere bundle $S^{*} \mathscr{F}$ to the foliation $\mathscr{F}$ of $M$ there is the following formula for the in $\mathrm{d}_{\nu}^{1}$ :

$$
\operatorname{ind}_{\nu}^{1}\left(\mathscr{D}, \rho_{0}, \rho_{1}, \varphi\right)=\left\langle\operatorname{Tch}_{\tau}\left(\nabla_{0}, \nabla_{1}\right) \operatorname{ch}_{\tau}\left(\pi_{+}(\sigma(D))\right) \operatorname{Todd}_{\tau}(M),\left[C_{\nu}\right]\right\rangle \text {. }
$$

The author would like to express his sincere gratitude to Professor H. Moscovici for valuable discussions and encouragement.

\section{Preliminaries}

In this section we collect the necessary facts about operators along the leaves of fcliation. For a thorough exposition, the reader is referred to [C1 and MS].

The holonomy groupoid. Let $(M, \mathscr{F})$ be a closed manifold with a foliation $\mathscr{F}$ of dimension $p$ (or codimension $q$ ) and $H: \pi_{1}(l, x) \rightarrow \mathrm{Homeo}_{0}\left(\mathbf{R}^{q}\right)$ a holonomy of a leaf $l$. A holonomy groupoid $G=G(M, \mathscr{F})$ is the quotient of the fundamental groupoid of $\mathscr{F}$ by the equivalence relation $\left[\gamma_{1}\right] \sim\left[\gamma_{2}\right]$ iff $\gamma_{1}(0)=\gamma_{2}(0), \gamma_{1}(1)=\gamma_{2}(1)$, and $H\left(\gamma_{2}^{-1} \gamma_{1}\right)=\mathrm{id} . G=G(M, \mathscr{F})$ is a (nonhausdorff) $(2 p+q)$-dimensional manifold. The canonical mappings $r, s: G \rightarrow M$,

\footnotetext{
${ }^{1}$ After this work was completed, it was brought to our attention that M. Ramachandran has independently proved the existence of eta invariant on foliated manifold.
} 
$r(x, y,[\gamma])=x, s(x, y,[\gamma])=y$, are open mappings and $s: G^{x} \rightarrow l(x)$ is a holonomy covering [W].

Let $\lambda=\left\{\lambda^{x}\right\}$ be a family of measures on $\left\{G^{x}\right\}$, each of which is obtained from a leafwise Riemannian metric such that the assignment $x \rightarrow G^{x}$ is measurable. If there exists a holonomy invariant transverse measure $\nu$ then $G$ becomes the measured groupoid $(G, \tilde{\mu})$, where

$$
\tilde{\mu}(E)=\int_{M} \lambda^{x}\left(E \cap G^{x}\right) d \mu(x) \text { and } \mu=\int d \nu .
$$

Moreover, the Hilbert space $L^{2}(G, \tilde{\mu})$ decomposes as a direct integral

$$
L^{2}(G, \tilde{\mu})=\int_{\oplus} L^{2}\left(G^{x}, \lambda^{x}\right) d \mu(x)
$$

The left regular representation of the measured groupoid $(G, \tilde{\mu})$ is the family $\left\{L_{\gamma}\right\}$ of isometries $L_{\gamma}: L^{2}\left(G^{s(\gamma)}, \lambda^{s(\gamma)}\right) \rightarrow L^{2}\left(G^{r(\gamma)}, \lambda^{r(\gamma)}\right)$ induced by the multiplication by $\gamma^{-1}$ from the left, $\gamma \in G$.

Foliation algebras. Let $C_{c}^{\infty, 0}(G)$ denote the space of all tangentially smooth functions of $G$ with compact support (here we assume that $G$ is Hausdorff). $C_{c}^{\infty, 0}(\Omega(G))$ is the *-algebra with involution, and for each $x \in M$ there is a natural representation $\pi_{x}$ of $C_{c}^{\infty, 0}(G)$ into the algebra of bounded operators on the Hilbert space $L^{2}\left(G^{x}, \lambda^{x}\right)$. It is given by

$$
\begin{aligned}
&\left(\left(\pi_{x} \varphi\right) \psi\right) \gamma=\int_{G^{x}} \varphi\left(\gamma^{-1} \gamma_{1}\right) \psi\left(\gamma_{1}\right) d \lambda^{x}\left(\gamma_{1}\right), \\
& \varphi \in C_{c}^{\infty, 0}(\Omega(G)), \psi \in L^{2}\left(G^{x}\right) .
\end{aligned}
$$

The reduced $C^{*}$ algebra of the groupoid $G(M, \mathscr{F}), C_{r}^{*}(G)$, is the completion of $C_{c}^{\infty, 0}(G)$ with respect to the norm $\|\varphi\|=\sup _{x \in M}\left\|\pi_{x}(\varphi)\right\|$.

The von Neumann algebra of a foliation, $W^{*}(G, \mathscr{F})$, can be characterized as a set of all operators $m$ on $L^{2}(G, \tilde{\mu})$ which have the direct integral decomposition $m=\int m_{x} d \nu$ and whose disintegration factors $m_{x}$ commute ( $\mu$ almost everywhere) with the left regular representation $\left\{L_{\gamma}\right\} . W^{*}(G, \mathscr{F})$ contains $C_{r}^{*}(G)$ as a dense subalgebra.

In the presence of a transverse measure $\nu$ there is a canonical faithful, semifinite normal trace $\Phi_{\nu}$ on $W^{*}(G, \mathscr{F})$. It is obtained essentially by integrating local traces against the transverse measure $\nu$.

Tangential cohomology. Let $\mathscr{R}_{\tau}$ be the sheaf of germs of tangentially locally constant real-valued functions on $M$ and let $\mathscr{R}$ be the sheaf of germs of locally constant real-valued functions on $M$. Then there is a short exact sequence of sheaves

$$
0 \rightarrow \mathscr{R} \rightarrow \mathscr{R}_{\tau} \rightarrow \mathscr{R} / \mathscr{R}_{\tau} \rightarrow 0 .
$$

The tangential cohomology $H_{\tau}^{*}(M, \mathbf{R})$ is defined as $H^{*}\left(M, \mathscr{R}_{\tau}\right)$, the cohomology of the sheaf $\mathscr{R}_{\tau} . H_{\tau}^{*}(M, \mathbf{R})$ is not necessarily Hausdorff as a topological vector space, the largest Hausdorff quotient of $H_{\tau}^{*}(M, \mathbf{R})$ is called a reduced tangential cohomology and denoted by $\bar{H}_{p}^{\tau}(M, \mathbf{R})$.

The sheaf map $\mathscr{R} \rightarrow \mathscr{R}_{\tau}$ induces a "restriction" map $r_{*}: H_{D R}^{*}(M, \mathbf{R}) \rightarrow$ $\bar{H}_{\tau}^{*}(M, \mathbf{R})$, and the tangential characteristic classes are by definition the images by $r_{*}$ of the "standard" characteristic classes, e.g., $\operatorname{Todd}_{\tau}(M, \mathscr{F})=r_{*} \operatorname{Todd}(M)$. 
The Riesz Representation Theorem (cf. [MS, pp. 128-132]) establishes an isomorphism between invariant transverse Radon measures $M T(M)$ and the top-dimensional tangential cohomology, $\bar{H}_{p}^{\tau}(M, \mathbf{R})$. For a given transverse measure $\nu$ and a tangentially smooth form $\omega$ it is defined by the pairing

$$
\langle\omega, \nu\rangle=\int_{M} \omega d \nu, \quad \omega \in \Omega_{\tau}^{p}(M) .
$$

Important for our applications will be the corollary that if the integrals of two $p$-dimensional tangential forms coincide for every invariant transverse Radon measure, then so do the forms as classes in $\bar{H}_{\tau}^{p}(M, \mathbf{R})$.

Tangential operators. A tangential $G$-operator is a family $\left(P_{x}\right)_{x \in M}$ of left $G_{x}^{x}$-invariant linear operators

$$
P_{x}: C^{\infty}\left(G^{x}, s^{*}\left(E_{1}\right)\right) \rightarrow C^{\infty}\left(G^{x}, s^{*}\left(E_{2}\right)\right) .
$$

Left invariance implies the existence of the vector-valued distribution $K$ on $G$ whose restriction $\left.K\right|_{G^{x}}=k$ satisfies $k\left(\gamma_{1}, \gamma_{2}\right)=K\left(\gamma_{1}^{-1} \gamma_{2}\right)$ so that

$$
P_{x}(\xi)\left(\gamma_{1}\right)=\int_{G^{x}} K\left(\gamma_{1}^{-1} \gamma_{2}\right) \xi\left(\gamma_{2}\right) d \lambda^{x}\left(\gamma_{2}\right)
$$

for every $\xi \in C_{c}^{\infty, 0}\left(s^{*}\left(E_{1}\right)\right)$ (in the weak sense). A tangential $G$-operator is called smoothing if its distributional kernel $K$ is of the class $C^{\infty, 0}$ on $\mathscr{F}(G)$. Examples of tangential $G$-operators are tangential differential operators obtained as follows. Let $D: \Gamma_{\tau}^{c o}\left(M, E_{1}\right) \rightarrow \Gamma_{\tau}^{\infty}\left(M, E_{2}\right)$ be a differential operator in which every foliation chart $\Omega \cong U \times V$ has the form $D_{\Omega}=$ $\sum_{|\alpha| \leq m} a_{\alpha}(x, y) D_{x}^{\alpha}$, where $a_{\alpha}(x, y)$ is of class $C^{\infty, 0}$ and $D_{x}^{\alpha}$ differentiates along the plaques. Let $\left\{\phi_{i}\right\}$ be a finite partition of unity subordinated to foliation charts. Then the lift of $D=\sum_{i}\left(\phi_{i} D\right)$ via the holonomy covering $s: G^{x} \rightarrow l(x)$ is clearly a tangential $G$-operator.

$D$ is said to be tangentially elliptic if its highest order symbol $\sigma(D)$ is invertible over the cosphere bundle $S^{*} \mathscr{F}$.

Proposition $0.1\left[\mathrm{C}_{1}\right]$. Let $\nu$ be an invariant transverse measure with associated trace $\Phi_{\nu}$ on $W^{*}(M, \mathscr{F})$. Then each tangential differential operator $D$ which has a continuous extension to

$$
W_{\tau}^{-s}(G, E) \rightarrow W_{\tau}^{s}(G, E)
$$

$(s>\operatorname{dim} \mathscr{F}=p)$ is in the domain of $\Phi_{\nu}$ and there is a constant $c$, independent of $D$, such that

$$
\left|\Phi_{\nu}(D)\right| \leq c\|D\|_{W_{\tau}^{-s}, W_{\tau}^{s}}
$$

In the sequel we will be using mostly Dirac-type operators as defined in [GL]. The letter $D$ will be reserved for arbitrary tangential differential operators while $\mathscr{D}$ will stand for the Dirac or Dirac-type tangential operators.

For the thorough discussion of the analysis on foliated manifolds the reader is referred to [C1], [C2] or [MS].

\section{The ETA INVARIANT}

Lemma 1.1. Let $\left\{A_{i}\right\}_{i \in I}$ be an increasing net of selfadjoint bounded operators on the field of Hilbert space $H_{i}$. Assume that the family $\left\{A_{i}\right\}_{i \in I}$ is bounded 
above by the operator $B: H \rightarrow H$. Then there exists a bounded and selfadjoint operator $A: H \rightarrow H$ such that

$$
A=\sup _{i \in I} A_{i} .
$$

Moreover, $A=\mathrm{s}-\lim _{i \in I} A_{i}$ (strong limit).

Proof. Standard. See [Y] for instance.

Lemma 1.2. Let $T: L^{2}(G, \mu) \rightarrow L^{2}(G, \mu)$ be an (unbounded) nonnegative selfadjoint operator. Let $f$ be a bounded Borel measurable function so that $f(T) \in W^{*}(G, \mu)$. Then

$$
\Phi_{\nu}(f(T))=\int_{0}^{\infty} f(x) d \alpha(x)
$$

where $\alpha=\Phi_{\nu} \lambda$ and $\lambda$ is a spectral resolution of $T$.

Proof. Suppose $\left\{B_{i}\right\}_{i=1}^{\infty}$ is a disjoint family of Borel subsets of $\mathbf{R}$. Then $\lambda\left(\bigcup_{i=1}^{n} B_{i}\right)$ is an increasing net of selfadjoint bounded operators, $\lambda\left(\bigcup_{i=1}^{n} B_{i}\right)$ is bounded above by $\lambda\left(\bigcup_{i=1}^{\infty} B_{i}\right) \leq \lambda(\mathbf{R})=1$. By Lemma 1.1 there exists $\sup _{n} \lambda\left(\bigcup_{i=1}^{n} B_{i}\right)$ and $\sup _{n} \lambda\left(\bigcup_{i=1}^{n} B_{i}\right)=\mathrm{s}-\lim _{n} \lambda\left(\bigcup_{i=1}^{n} B_{i}\right)$. Thus $\lambda\left(\bigcup_{i=1}^{n} B_{i}\right)=$ $\sup _{n} \lambda\left(\bigcup_{i=1}^{n} B_{i}\right)$ by the strong continuity of the spectral measure $\lambda$. By the normality of $\Phi_{\nu}$, we have

$$
\Phi_{\nu} \lambda\left(\bigcup_{i=1}^{\infty} B_{i}\right)=\Phi_{\nu} \sup _{n} \lambda\left(\bigcup_{i=1}^{n} B_{i}\right)=\sup _{n} \Phi_{\nu} \lambda\left(\bigcup_{i=1}^{n} B_{i}\right)=\bigcup_{i=1}^{\infty} \Phi_{\nu} \lambda\left(B_{i}\right)
$$

what proves $\sigma$-additivity of $\nu=\Phi_{\nu} \lambda$.

The proof of the equality (1.2) is similar. One starts with simple functions and relies on Lemma 1.1. This completes the proof.

Let $D: \Gamma_{\tau}^{\infty}(M, E) \rightarrow \Gamma_{\tau}^{\infty}(M, E)$ be a tangential tangentially elliptic differential operator. The following is motivated by the estimate in [CG]:

Proposition 1.3. For every $s \in \mathbf{C}, \operatorname{Re}(s) \leq 0$, the integral

$$
\int_{1}^{\infty} t^{(s-1) / 2} \Phi_{\nu}\left(D e^{-t D^{2}}\right) d t
$$

is convergent.

Proof. $\left|t^{(s-1) / 2}\right| \leq t^{(\operatorname{Re}(s)-1) / 2} \leq t^{-1 / 2}, 0 \leq t \leq \infty$, implies

$$
\begin{gathered}
\int_{1}^{\infty}\left|t^{(s-1) / 2} \Phi_{\nu}\left(D e^{-t D^{2}}\right) d t\right| \leq \int_{1}^{\infty}\left|t^{(s-1) / 2}\right|\left|\Phi_{\nu}\left(D e^{-t D^{2}}\right)\right| d t \\
\quad \leq \int_{1}^{\infty} t^{-1 / 2}\left|\Phi_{\nu}\left(D e^{-t D^{2}}\right)\right| d t \leq \int_{1}^{\infty} t^{-1 / 2} \Phi_{\nu}\left(|D| e^{-t D^{2}}\right) d t .
\end{gathered}
$$

Now Lemma 1.2 applied to the operator $T=D^{2}=|D|^{2}$ gives the existence of the Borel measure $\mu$ on $\mathbf{R}$ such that the last integral is equal to

$$
\int_{1}^{\infty} t^{-1 / 2} d t \int_{0}^{\infty} \lambda^{1 / 2} e^{-t \lambda} d \mu(\lambda)
$$


We estimate

$$
\begin{aligned}
\int_{0}^{\infty} & \lambda^{1 / 2} d \mu(\lambda) \int_{1}^{\infty} t^{-1 / 2} e^{-t \lambda} d t \\
& =\int_{0}^{\infty} \lambda^{1 / 2} e^{-\lambda} d \mu(\lambda) \int_{1}^{\infty} t^{-1 / 2} e^{-\lambda(t-1)} d t \\
& =\int_{0}^{\infty} e^{-\lambda} d \mu(\lambda) \int_{0}^{\infty}(u+\lambda)^{-1 / 2} e^{-u} d u \\
& \leq \int_{0}^{\infty} e^{-\lambda} d \mu(\lambda) \int_{0}^{\infty} u^{-1 / 2} e^{-u} d u \\
& =\pi^{1 / 2} \int_{0}^{\infty} e^{-\lambda} d \mu(\lambda)=\pi^{1 / 2} \Phi_{\nu}\left(e^{-D^{2}}\right)<\infty !
\end{aligned}
$$

Proposition 1.4. Let $(M, \mathscr{F})$ be a closed foliated space and $\mathscr{D}: \Gamma_{\tau}^{\infty}(M, S) \rightarrow$ $\Gamma_{\tau}^{\infty}(M, S)$ a Dirac-type operator. Then there exists a family of tangentially smooth and locally computable functions $\left\{\Psi_{m}\right\}_{m \geq 0}$ so that the kernel $K_{t}(x, y, n)$ of the operator $\mathscr{D} \exp \left(-t \mathscr{D}^{2}\right)$ has the asymptotic expansion

$$
K_{t}(x, x, n) \approx \sum_{m \geq 0} t^{(m-p-1) / 2} \Psi_{m}(x, n)
$$

where $p=\operatorname{dim} \mathscr{F}$. Moreover, the remainder terms implicit in the expansion are uniformly bounded on $M$.

Proof. This is a modification of a classical proof about heat kernel asymptotics (see, e.g., [R]). It uses a bounded geometry of leaves and is explained in [P1].

Corollary 1.5. $\Psi_{m}$ are locally bounded tangential measures, $\Psi_{m} \equiv 0$ if $m$ is even.

Proof. The proof uses Gilkey's invariance theory and explicit form of $\Psi_{m}$. See [P1] for details.

Using the fact that the trace of an operator is the integral of its kernel, from Proposition 1.4 and Corollary 1.5 we obtain

$$
\begin{gathered}
\Phi_{\nu}\left(\mathscr{D} \exp \left(-t \mathscr{D}^{2}\right)\right) \approx \sum_{m \geq 0} t^{(m-p-2) / 2} \int_{M} \Psi_{m}(\mathscr{D}) d \nu \\
\frac{1}{\Gamma(s+1) / 2} \int_{0}^{1} t^{(s-1) / 2} \Phi_{\nu}\left(\mathscr{D} \exp \left(-t \mathscr{D}^{2}\right)\right) \\
\approx \sum_{m \geq 0} \frac{2}{s+m-p} \int_{M} \Psi_{m}(\mathscr{D}) d \nu,
\end{gathered}
$$

where $\Gamma$ is the Gamma function and $\operatorname{Re}(s) \leq 0$.

\section{Definition 1.6.}

$$
\eta(\mathscr{D}, s)=\frac{1}{\Gamma((s+1) / 2)} \int_{0}^{\infty} t^{(s-1) / 2} \Phi_{\nu}\left(\mathscr{D} \exp \left(-t \mathscr{D}^{2}\right)\right) d t, \quad \operatorname{Re}(s) \leq 0 .
$$

Thus from Proposition 1.3, Corollary 1.5 and (1.12) we see that $\eta(\mathscr{D}, s)$ is a well-defined and meromorphic function with eventually simple poles at $(\operatorname{dim} \mathscr{F}-k) / 2(k=0,1,2, \ldots)$.

Next we show that the eta function of a Dirac operator on an even-dimensional foliation vanishes. 
Lemma 1.7. If $\operatorname{dim} \mathscr{F}=p$ is even then the Dirac operator $\mathscr{D}: L^{2}(G, \lambda) \rightarrow$ $L^{2}(G, \lambda)$ has a symmetric spectrum with respect to the origin.

Proof. Indeed, for $p$ even one defines a "volume form" $\omega \in \Gamma_{\tau}\left(\operatorname{Cliff}\left(T^{*} \mathscr{F}\right)\right)$ locally representable as

$$
\omega=i^{p / 2} e_{1} e_{2} \cdots e_{m}
$$

where $i=\sqrt{-1}$ and $\left\{e_{1}, e_{2}, \ldots, e_{m}\right\}$ is a local orthonormal basis of $T^{*} \mathscr{F}$. $\omega$ has the following properties:

$$
\omega^{2}=1, \quad \nabla \omega=0, \quad \omega e=-e \omega
$$

where $\nabla$ is the connection on $\operatorname{Cliff}\left(T^{*} \mathscr{F}\right)$ induced by the tangential Levi-Civita connection on $T \mathscr{F}$, and $e \in T^{*} \mathscr{F}$ is a unit vector (see [LM]). From (1.14) it follows that there exists an orthogonal decomposition of $S=S^{+} \oplus S^{-}$into the \pm 1 eigenbundles of $\omega$. In fact $S^{ \pm}=(1 \pm \omega) S$. $\mathscr{D}$ switches the (sections of ) eigenbundles $S^{ \pm}$and gives rise to a pair of operators $\mathscr{D}^{ \pm}: \Gamma_{\tau}\left(S^{ \pm}\right) \rightarrow \Gamma_{\tau}\left(S^{\mp}\right)$,

$$
\mathscr{D}=\left[\begin{array}{cc}
0 & \mathscr{D}^{-} \\
\mathscr{D}^{+} & 0
\end{array}\right]
$$

It can be checked that the decompositions above yield to

$$
L_{\tau}^{2}(M, S)=L_{\tau}^{2}\left(M, S^{+}\right) \oplus L_{\tau}^{2}\left(M, S^{-}\right)
$$

and an analogous decomposition for $D$. Clearly the same holds for each holonomy covering $G^{x}$ and each $\mathscr{D}_{x}$, the lifting of $\mathscr{D}_{l}$ to $G^{x}, x \in l$. In short, we can write $L^{2}\left(G^{x}, s^{*}(S)\right)=L^{2}\left(G^{x}, s^{*}\left(S^{+}\right)\right) \oplus L^{2}\left(G^{x}, s^{*}\left(S^{-}\right)\right)$and

$$
\mathscr{D}_{x}=\left[\begin{array}{cc}
0 & \mathscr{D}_{x}^{-} \\
\mathscr{D}_{x}^{+} & 0
\end{array}\right]
$$

for every $x \in M$ and every $l \in M / \mathscr{F}, x \in l$. Now it can be easily seen that $\left[\begin{array}{cc}0 & 1 \\ -1 & 0\end{array}\right]$ is the unitary equivalence between $\mathscr{D}_{x}$ and $-\mathscr{D}_{x}$ and, moreover, that

$$
\left[\begin{array}{cc}
0 & 1 \\
-1 & 0
\end{array}\right] P_{x}\left[\begin{array}{cc}
0 & 1 \\
-1 & 0
\end{array}\right]^{-1}=-P_{x}
$$

for every spectral projection $P_{x}$ of $\mathscr{D}_{x}, x \in M$. This completes the proof.

Corollary 1.8. If $\operatorname{dim} \mathscr{F}$ is even then $\eta(\mathscr{D}, s)=0$ for every $s \in \mathbf{C}, \operatorname{Re}(s) \leq 0$. Proof. Lemma 1.7 implies that $\mathscr{D} \exp \left(-t \mathscr{D}^{2}\right)$ has a symmetric spectrum as well, so by Lemma $1.2, \Phi_{\nu}\left(\mathscr{D} \exp \left(-t \mathscr{D}^{2}\right)\right)=0$. Hence the result.

When $\operatorname{dim} \mathscr{F}$ is odd then the regularity of the eta function at the origin follows from the deep result of Bismut and Fried [BF]. They show that the ordinary local trace of a Dirac operator satisfies $\operatorname{tr}\left(\mathscr{D} \exp \left(-t \mathscr{D}^{2}\right)\right)=O\left(t^{1 / 2}\right)$, $t \downarrow 0$. Since $\nu$ - trace can be locally approximated by the regular trace (cf. [C1] their result applies to our setting to give

$$
K_{t}(x, x, n) \sim \sum_{m \geq p+2} t^{(m-p-1) / 2} \Psi_{\text {a.e. }}(x, n) .
$$

Together with (1.12) this implies the regularity of the $\eta$ function at the origin. We collect previously obtained results in 
Theorem 1.9. Let $\mathscr{D}: \Gamma_{\tau}^{\infty}(M, S) \rightarrow \Gamma_{\tau}^{\infty}(M, S)$ be a tangential Dirac-type operator on the foliated manifold $M$. Then its eta function,

$$
\eta(\mathscr{D}, s)=\Phi_{\nu}\left(\mathscr{D} \exp \left(-t \mathscr{D}^{2}\right)\right)
$$

is a well-defined and meromorphic function for $\operatorname{Re}(s) \leq 0$ with eventually simple poles at $(\operatorname{dim} \mathscr{F}-k) / 2(k=0,1,2, \ldots) . \eta(\mathscr{D}, s)$ is regular at $s=0$.

However, if the restriction of the operator $D$ to $\nu$-almost all leaves is invertible, then we can prove a stronger statement than in Proposition 1.3 and Theorem 1.9.

Lemma 1.10. If $D: \Gamma_{\tau}^{\infty}(M, S) \rightarrow \Gamma_{\tau}^{\infty}(M, S)$ is ( $\nu$-a.e.) invertible then the integral $\int_{0}^{\infty} t^{(s-1) / 2} \Phi_{\nu}\left(D \exp \left(-t D^{2}\right)\right) d t$ is analytic in $s \in \mathbf{C}$ and $\eta(D, s)$ extends to the whole complex plane.

Proof. $0 \notin \sigma(D)$ implies that $D^{-k+1}$ exists and by Proposition $0.1, D^{-k+1} \in$ $\operatorname{Dom}\left(\Phi_{\nu}\right)$ for $k$ large enough. By Proposition 1.2,

$$
\int_{-\infty}^{\infty} x^{-k+1} d \alpha(x)<\infty, \quad \alpha=\Phi_{\nu} \lambda
$$

where $\lambda$ denotes the spectral measure of $D$. Now

$$
\begin{aligned}
t^{(s-1) / 2} \Phi_{\nu}\left(D e^{-t D^{2}}\right) & =\int_{-\infty}^{\infty} t^{(s-1) / 2} x e^{-t x^{2}} d \alpha(x) \\
& =\int_{-\infty}^{\infty} t^{(s-1) / 2} x^{k} e^{-t x^{2}}\left(x^{-k+1} d \alpha(x)\right) .
\end{aligned}
$$

It can be easily checked that $\varphi(x)=x^{k} e^{-t x^{2}}$ satisfies $\varphi(x) \leq c_{k} t^{-k / 2}, c_{k}$ being independent of $x$, hence

$$
\begin{aligned}
t^{(s-1) / 2} \Phi_{\nu}\left(D e^{-t D^{2}}\right) & \leq c_{k} \int_{-\infty}^{\infty} t^{(s-k-1) / 2} x^{-k+1} d \alpha(x) \\
& =\tilde{c}_{k} t^{(s-k-1) / 2}
\end{aligned}
$$

Therefore, given $s \in \mathbf{C}$, then

$$
\int_{1}^{\infty} t^{(s-1) / 2} \Phi_{\nu}\left(D e^{-t D^{2}}\right) d t<\infty
$$

since $k$ can be chosen to be arbitrarily large. The integral

$$
\int_{1}^{\infty} t^{(s-1) / 2} \Phi_{\nu}\left(D e^{-t D^{2}}\right) d t
$$

is uniformly convergent in $s ; t^{(s-1) / 2} \Phi_{\nu}\left(D e^{-t D^{2}}\right)$ is continuous in $t$ and uniformly bounded in $s$ by an integrable function. It follows that

$$
s \rightarrow \int_{1}^{\infty} t^{(s-1) / 2} \Phi_{\nu}\left(D e^{-t D^{2}}\right) d t
$$

is an entire function (see, e.g., [E]). 
Proposition 1.11. Let $\mathscr{D}_{u}: \Gamma_{\tau}^{\infty}(M, S) \rightarrow \Gamma_{\tau}^{\infty}(M, S), 0 \leq u \leq 1$, be a smooth, one-parameter family of ( $\nu$-a.e.) invertible Dirac-type operators. Then,

$$
\begin{aligned}
\frac{d \eta\left(\mathscr{D}_{u}, s\right)}{d u} & =-s \Phi_{\nu}\left(\mathscr{D}_{u}\left|\mathscr{D}_{u}\right|^{-s-1}\right) \\
& \sim \frac{-s}{\Gamma((s+1) / 2)} \sum_{n=0}^{\infty} \frac{2}{s+n-p} a_{n}\left(\mathscr{D}_{u}, \mathscr{D}_{u}^{2}\right), \quad \operatorname{Re}(s) \gg 0
\end{aligned}
$$

and

$$
\frac{d \eta\left(\mathscr{D}_{u}, 0\right)}{d u} \sim \frac{-1}{\Gamma\left(\frac{1}{2}\right)} a_{p}\left(\mathscr{D}_{u}, \mathscr{D}_{u}^{2}\right)
$$

where $\mathscr{D}_{u}$ denotes differentiation with respect to $u$ and $a_{p}\left(\mathscr{D}_{u}, \mathscr{D}_{u}^{2}\right)$ is a local invariant obtained by integrating certain tangential measure against transverse measure.

We only remark that the condition $\operatorname{Re}(s) \gg 0$ under (a) is possible because of the analyticity of the tail $\int_{1}^{\infty} t^{(s-1) / 2} \Phi_{\nu}\left(\mathscr{D} \exp \left(-t \mathscr{D}^{2}\right)\right) d t$. The proof of Proposition 1.11 will be omitted. By using Lemma 1.14 it can be obtained as a modification of a known proof (cf. [APS] and [P1]). Before we proceed to the proof of the index theorem we will explain how the Dirac and boundary Dirac operators are related.

Suppose $N=M \times[0,1)$ is a spin manifold, $\operatorname{dim} N=2 n, \mathscr{D}: \Gamma_{\tau}^{\infty}\left(S^{+}\right) \rightarrow$ $\Gamma_{\tau}^{\infty}\left(S^{-}\right)$is a half Dirac operator, and $S^{+}, S^{-}$are spinor bundles associated to irreducible representations of $\operatorname{Spin}(2 n)$. When the metric on the collar neighborhood of $M \times\{0\} \subset N$ is a product metric, one can write

$$
\mathscr{D}^{+}=c(d(n))\left(\frac{\partial}{\partial n}+A^{+}\right)
$$

where $c(d(n))$ is a Clifford multiplication by unit normal $d n, n$ inward coordinate on $[0,1)$. The operator $c(d(n))$ switches $S^{ \pm}$to $S^{\mp}$ thus $A^{+}$acts as $A^{+}: \Gamma_{\tau}^{\infty}\left(S^{+}\right) \rightarrow \Gamma_{\tau}^{\infty}\left(S^{+}\right)$. $A^{+}$is called the boundary Dirac operator.

As $c(d(n))^{2}=-1$, it follows that $\sigma\left(A^{+}\right) \xi=-c(d(n)) c(\xi), \xi \in T^{*} M .\left.S^{+}\right|_{M}$ can be identified with the spinor bundle of $M, S(M)$, so $A^{+}: \Gamma_{\tau}^{\infty}(S(M)) \rightarrow$ $\Gamma_{\tau}^{\infty}(S(M))$. Define volume elements $\omega_{2 n}=i^{n} e_{1} \cdots e_{2 n}$ and $\omega_{2 n-1}=i^{n} e_{1} \cdots$ $e_{2 n-1}$ and the isomorphism $\tau_{+}:\left.S(M) \rightarrow S^{+}\right|_{M}, \tau_{+}(\phi)=\phi+\omega_{2 n} \phi$. We claim that the following diagram is commutative:

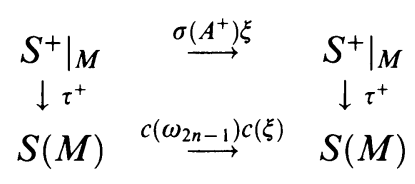


Proof. Let $\varphi \in S(M), \xi \in T^{*}(M)$. Then

$$
\begin{aligned}
\tau_{+}^{-1}( & -c(d(n)) c(\xi)) \tau_{+} \varphi=\tau_{+}^{-1}(-c(d(n)) c(\xi))\left(\varphi+c\left(\omega_{2 n}\right) \varphi\right) \\
= & \tau_{+}^{-1}\left(-c(d(n)) c(\xi) \varphi-c(d(n)) c(\xi) c\left(\omega_{2 n}\right) \varphi\right) \\
= & \tau_{+}^{-1}\left(-c(d(n)) c(\xi) \varphi-c(d(n)) c(\xi) c(-d(n)) c\left(\omega_{2 n-1}\right) \varphi\right) \\
= & \tau_{+}^{-1}\left(-c(d(n)) c(\xi) \varphi+c(\xi) c\left(\omega_{2 n-1}\right) \varphi\right) \\
& \left(c\left(\omega_{2 n-1}\right)\right. \text { is the central element in } \\
& \operatorname{Cl}(T M) \text { since dim } M \text { is odd }) \\
= & \tau_{+}^{-1}\left(-c(d(n)) c(\xi) \varphi+c\left(\omega_{2 n-1}\right) c(\xi) \varphi\right) \\
= & \tau_{+}^{-1}\left(-c(d(n)) c\left(\omega_{2 n-1}\right) c\left(\omega_{2 n-1}\right) c(\xi) \varphi+c\left(\omega_{2 n-1}\right) c(\xi) \varphi\right) \\
= & \tau_{+}^{-1}\left(c\left(\omega_{2 n}\right)+1\right)\left(c\left(\omega_{2 n-1}\right) c(\xi)\right) \varphi=c\left(\omega_{2 n-1}\right) c(\xi) \varphi
\end{aligned}
$$

Lemma 1.12. Let $A^{+}$be the boundary Dirac operator,

$$
A^{+}: \Gamma_{\tau}^{\infty}\left(\left.S^{+}\right|_{M}\right) \rightarrow \Gamma_{\tau}^{\infty}\left(\left.S^{+}\right|_{M}\right)
$$

Then $A^{+}$is naturally isomorphic to the operator

$$
\Gamma_{\tau}^{\infty}(S(M)) \stackrel{\nabla}{\rightarrow} \Gamma_{\tau}^{\infty}\left(T^{*} M \otimes S(M)\right) \stackrel{c\left(\omega_{2 n-1}\right) c(\xi)}{\rightarrow} \Gamma_{\tau}^{\infty}(S(M))
$$

Proof. We have proved it already on the level of symbols. The Levi-Civita connection on $N=M \times[0,1)$ is just the flat extension of the Levi-Civita connection from $M$. Since $\omega_{2 n-1}, \omega_{2 n}$ are globally parallel it follows also that the second square

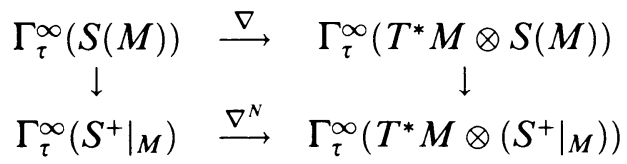

is also commutative. This completes the proof of the lemma.

\section{A genERALIZATION OF THE RELATIVE INDEX THEOREM}

Recall the setting for the relative index theorem. Given the two unitary representations of the fundamental group of $M$, say $\rho_{i}: \pi_{1}(M) \rightarrow U(n) \quad(i=$ $0,1)$, one forms flat bundles $V_{i}=\widetilde{M}_{\rho_{i}} \times \mathbf{C}^{n} \quad(\widetilde{M}$ is the universal covering of $M)$. These bundles have discrete structure groups and inherited flat connections $\nabla_{i}(i=0,1)$. Then $\mathscr{D}: \Gamma_{\tau}^{\infty}(M, S \otimes E) \rightarrow \Gamma_{\tau}^{\infty}(M, S \otimes E)$ can be extended to operators $\mathscr{D}_{i}: \Gamma_{\tau}^{\infty}\left(M, S \otimes E \otimes V_{i}\right) \rightarrow \Gamma_{\tau}^{\infty}\left(M, S \otimes E \otimes V_{i}\right) \quad(i=0,1)$ so that each $\mathscr{D}_{i}$ is locally isomorphic to $\mathscr{D} \otimes I$; in particular, $\mathscr{D}_{i}$ 's have the same principal symbol. If, besides the representations $\rho_{i}$, additional secondary data is given, namely an isomorphism $\varphi$ of bundles $V_{0}$ and $V_{1}$, then we can regard operators $\mathscr{D}_{i}$ as defined on the same bundle (replacing $\mathscr{D}_{1}$ by $\varphi^{-1} \mathscr{D}_{1} \varphi$ ) and define the family $\mathscr{D}_{u}=(1-u) \mathscr{D}_{0}+u \mathscr{D}_{1} \quad(0 \leq u \leq 1)$ of operators on $\Gamma_{\tau}^{\infty}(M, S \otimes E \otimes V)$, $V=V_{0}$.

Theorem 2.1. Let $(M, \mathscr{F})$ be a foliated spin manifold of odd dimension $p$. Let $S \rightarrow M$ be a corresponding tangential spin bundle and $E \rightarrow M$ a coefficient bundle. Let $\mathscr{D}_{u}: \Gamma_{\tau}^{\infty}(M, S \otimes E) \rightarrow \Gamma_{\tau}^{\infty}(M, S \otimes E), 0 \leq u \leq 1$, be a family of 
invertible Dirac operators associated with the family $\nabla_{u}^{E}$ of connections on the bundle $E$ and the spinor connection $\nabla^{S}$. Then

$$
\left.\frac{d \eta\left(\mathscr{D}_{u}, s\right)}{d u}\right|_{s=0}=\int_{M} a_{p}\left(x, \dot{\mathscr{D}}_{u}, \mathscr{D}_{u}^{2}\right) d \nu
$$

where

$$
a_{p}\left(x, \dot{\mathscr{D}}_{u}, \mathscr{D}_{u}^{2}\right)=\sum_{4 i+2 j=p-1}\left(\frac{\sqrt{-1}}{2 \pi}\right)^{j} \frac{1}{(j-1) !} \widehat{A}_{\tau, i} \cap \operatorname{tr}\left(\psi \cap \Omega_{E}^{j-1}\right)
$$

as a class in $\bar{H}_{\tau}^{p}(M, \mathscr{F}) . \Omega_{E}=\Omega_{E}(u)$ is the curvature of $\nabla^{S} \otimes \nabla_{u}^{E}$ and $\psi$ is a 1 -form $\nabla^{S} \otimes \nabla_{1}^{E}-\nabla^{S} \otimes \nabla_{0}^{E}$.

Proof. According to Proposition 1.11, $d \eta\left(\mathscr{D}_{u}, s\right) /\left.d u\right|_{s=0}$ is given by the integral of some tangential measure $a_{p}$ against the transverse measure $\nu$. A change of orientation of the tangential bundle of $\mathscr{F}$ yields a sign change of $a_{p}$. So $a_{p}$ must be a tangentially smooth form. Restrict $a_{p}$ to a leaf $l$. It is proved in the appendix that the restriction $\left.a_{p}\right|_{l}$ of $a_{p}$ to the leaf $l$ is equal to the right-hand side of (2.2). Hence the integral of $a_{p}$ with respect to any Radon invariant transverse measure $\nu$ coincides with the integral of the right-hand side of (2.2). By the Corollary of the Hahn-Banach Theorem (see (0.4) or [MS, pp. 128-132]) it follows that

$$
a_{p}\left(x, \mathscr{D}, \rho_{1}, \rho_{2}\right)=\sum_{4 u+2 v=p+1}\left(\frac{\sqrt{-1}}{2 \pi}\right)^{v} \frac{1}{(v-1) !} \widehat{A}_{\tau}(g) \cap \operatorname{tr}\left(\psi \cap \Omega_{E \otimes V}^{v-1}\right)
$$

as classes in $\bar{H}_{\tau}^{p}(M, \mathscr{F})$. Hence the result.

If we assume that each $\mathscr{D}_{u}$ is invertible then $d \eta\left(\mathscr{D}_{u}, s\right) / d u=d \eta(u, s) / d u$ is a well-defined $\mathbf{R}$-valued function. The variation

$$
\begin{aligned}
\eta\left(\mathscr{D}_{2}, 0\right)-\eta\left(\mathscr{D}_{1}, 0\right) & =\left.\int_{0}^{1} \frac{d \eta(u, s)}{d u}\right|_{s=0} d u \\
& =\int_{M} d \nu \int_{0}^{1} a_{p}\left(x, \dot{\mathscr{D}}_{u}, \mathscr{D}_{u}^{2}\right) d u \\
& =\int_{M} a_{p}(x, \mathscr{D}) d \nu
\end{aligned}
$$

is called the relative index of $\mathscr{D}$ and denoted by $\operatorname{ind}_{\nu}^{1}\left(\rho_{0}, \rho_{1}, \varphi, \mathscr{D}\right)$.

Next we show that under the described conditions we get a generalization of the Atiyah-Patodi-Singer relative index theorem.

\section{Theorem 2.2.}

$$
\operatorname{ind}_{\nu}^{1}\left(\rho_{0}, \rho_{2}, \varphi, D\right)=\left\langle\operatorname{Tch}_{\tau}\left(\nabla_{0}^{V}, \nabla_{1}^{V}\right) \operatorname{ch}_{\tau}\left(\pi_{+}(\sigma(\mathscr{D}))\right) \operatorname{Todd}_{\tau}(M),\left[C_{\nu}\right]\right\rangle
$$

where $\left[C_{\nu}\right]$ is the Ruelle-Sullivan class.

Lemma 2.3. Let $E \rightarrow M$ be an $n$-dimensional tangentially smooth vector bundle over the compact foliated manifold $M$. Then there exists, up to a constant, $a$ unique mapping $I_{\tau}: \Omega_{\tau c}^{*}(E) \rightarrow \Omega_{\tau}^{*}(M)$ from the tangentially smooth compactly 
supported forms on $E$ to the tangentially smooth forms on $M$ satisfying the following properties:

(1) $I_{\tau}$ is a $\Omega_{\tau}^{*}(M)$ module homomorphism, i.e.,

$$
I_{\tau}\left(\pi^{*}(\beta) \cap \alpha\right)=\beta \cap I_{\tau}(\alpha), \quad \alpha \in \Omega_{\tau c}^{*}(E), \beta \in \Omega_{\tau}^{*}(M) .
$$

(2) $\int_{E} \alpha d \mu=\int_{M} I_{\tau}(\alpha) d \nu, \alpha \in \Omega_{\tau c}^{*}(E)$.

Here $d \mu$ is the holonomy invariant measure on the foliation of $E$ induced by the measure $\nu$.

The proof of Lemma 2.3 is a straightforward generalization of the "integration along the fibre."

Lemma 2.4. If $p=\operatorname{dim} \mathscr{F}$ and $4 i=p-2 j+1, i, j \in \mathbf{N}$, then

$$
\left\{I_{\tau}\left(\operatorname{ch}^{\tau}\left(\pi_{+}(\sigma(\mathscr{D}))\right) \operatorname{Todd}_{\tau}(M)\right\}_{p-2 j+1}=\widehat{A}_{i}^{\tau}(M)\right.
$$

in $\bar{H}_{\tau}^{p}(M, \mathscr{F})$. Here $\widehat{A}^{\tau}(M)$ is the tangential A-roof genus of $M$.

Proof. This is a fairly involved topological calculation. A proof may be found in [Sh].

Proof of Theorem 2.2. We claim that

$$
\begin{aligned}
& \operatorname{ind}^{1}\left(\mathscr{D}_{E}, \rho_{1}, \rho_{0}\right)=\eta\left(\mathscr{D}_{1, E}, 0\right)-\eta\left(\mathscr{D}_{0, E}, 0\right) \\
& \quad=\int_{S(\mathscr{F})} \operatorname{Tch}^{\tau}\left(\nabla_{0}^{V}, \nabla_{1}^{V}, \rho\right) \operatorname{ch}^{\tau}\left(\sigma\left(\pi_{+}\left(\mathscr{D}_{E}\right)\right)\right) \operatorname{Todd}_{\tau}(M) d \mu .
\end{aligned}
$$

From Theorem 2.1 we have

$$
\begin{aligned}
\left.\frac{d \eta(u, s)}{d u}\right|_{s=0} & =a_{p}\left(x, \dot{\mathscr{D}}_{u}, \mathscr{D}_{u}^{2}\right) \\
& =\sum_{4 i+2 j=p-1}\left(\frac{\sqrt{-1}}{2 \pi}\right)^{j} \frac{1}{(j-1) !} \widehat{A}_{\tau, i} \cap \operatorname{tr}\left(\psi \cap \Omega_{E}^{j-1}\right)
\end{aligned}
$$

where $\psi$ is a connection 1 -form of

$$
\frac{d}{d u} \nabla_{u}^{E \otimes V}=\frac{d}{d u}\left(\nabla^{E} \otimes I+I \otimes \nabla_{u}^{V}\right)=I \otimes \psi^{1}
$$

and $\psi^{1}$ is the connection 1 -form of

$$
\frac{d}{d u} \nabla_{u}^{V}=\frac{d}{d u}\left[(1-u) \nabla_{0}^{V}+u \nabla_{1}^{V}\right]=\nabla_{1}^{V}-\nabla_{0}^{V} .
$$

Also, $\Omega_{E \otimes V}$ is a derivation, $\Omega_{E \otimes V}=\Omega_{E} \otimes I+I \otimes \Omega_{V}$. Consequently,

$$
\begin{aligned}
\operatorname{tr}\left(\psi \cap \Omega_{E \otimes V}^{j-1}\right) & =\operatorname{tr}\left[I \otimes \psi^{1}\left(\Omega_{E} \otimes I+I \otimes \Omega_{V}\right)^{j-1}\right] \\
& =\sum_{q+r=j-1} \frac{(j-1) !}{q ! r !} \operatorname{tr}\left(\Omega_{E}^{q}\right) \operatorname{tr}\left(\psi^{1} \cap \Omega_{V}^{r}\right) .
\end{aligned}
$$

Consequently,

$$
\begin{aligned}
& \left(\frac{\sqrt{-1}}{2 \pi}\right)^{j} \frac{1}{(j-1) !} \operatorname{tr}\left(\psi \cap \Omega_{E \otimes V}^{j-1}\right) \\
& \quad=\left\{\operatorname{ch}^{\tau}(E) \sum_{r}\left(\frac{\sqrt{-1}}{2 \pi}\right)^{r} \frac{1}{(r-1) !} \operatorname{tr}\left(\psi \cap \Omega_{V}^{r-1}\right)\right\}_{2 j-1} .
\end{aligned}
$$


Thus, $d \eta(u, s) /\left.d u\right|_{s=0}$ equals

$$
\int_{M} \sum_{4 i+2 j-1=p}\left\{\operatorname{ch}^{\tau}(E) \sum_{r}\left(\frac{\sqrt{-1}}{2 \pi}\right)^{r} \frac{1}{(r-1) !} \operatorname{tr}\left(\psi \cap \Omega_{V}^{r-1}\right)\right\}_{2 j-1} \widehat{A}_{i}^{\tau} .
$$

So, from (2.13) and Lemma 2.4 we have $d \eta\left(\mathscr{D}_{E \otimes V, u}, s\right) /\left.d u\right|_{s=0}$ equals

$$
\int_{M} \sum_{r \geq 0}\left(\frac{1}{2 \pi}\right)^{r} \frac{1}{(r-1) !} \operatorname{tr}\left(\psi \cap \Omega_{V}^{r-1}\right) \operatorname{ch}^{\tau}(E)\left(I_{\tau}\left(\operatorname{ch}^{\tau}\left(\pi_{+}(\sigma)\right)\right) \operatorname{Todd}_{\tau}(M) d v\right.
$$

and, by the $\Omega_{\tau}^{*}(M)$ linearity of $I_{\tau}$,

$$
\begin{aligned}
&=\int_{M} \sum_{r \geq 0}\left(\frac{1}{2 \pi}\right)^{r} \frac{1}{(r-1) !} \operatorname{tr}\left(\psi \cap \Omega_{V}^{r-1}\right) \\
& \cdot I_{\tau}\left[\operatorname{ch}^{\tau}(E)\left(\operatorname{ch}^{\tau}(E)\left(\operatorname{ch}^{\tau}\left(\pi_{+}(\sigma)\right)\right)\right] \operatorname{Todd}_{\tau}(M) d v\right.
\end{aligned}
$$

$$
=\int_{S(\mathscr{F})} \sum_{r \geq 0}\left(\frac{1}{2 \pi}\right)^{r} \frac{1}{(r-1) !} \operatorname{tr}\left(\psi \cap \Omega_{V}^{r-1}\right) \operatorname{ch}^{\tau}\left(\pi_{+}\left(\sigma\left(\mathscr{D}_{E}\right)\right)\right) \operatorname{Todd}_{\tau}(M) d \mu .
$$

Now we use the assumption that $\nabla_{0}$ and $\nabla_{1}$ are flat. It can be easily shown that the curvature $\Omega_{V}=\Omega_{V, u}$ satisfies $\Omega_{V}=\left(u-u^{2}\right) \psi^{1} \wedge \psi^{1}$. Thus integrating the above equality with respect to $u$ and taking into account that for the flat tangential connections $\nabla_{0}$ and $\nabla_{1}, d \operatorname{Tch}_{k}^{\tau}\left(\nabla_{0}, \nabla_{1}\right)=\operatorname{ch}_{k}^{\tau}\left(\nabla_{1}\right)-\operatorname{ch}_{k}^{\tau}\left(\nabla_{0}\right)$,

$$
\begin{aligned}
& \eta\left(\mathscr{D}_{1, E}, 0\right)-\eta\left(\mathscr{D}_{0, E}, 0\right)=\operatorname{ind}^{1}\left(\mathscr{D}_{E}, \rho_{1}, \rho_{0}\right) \\
& \quad=\int_{S(\mathscr{F})} \operatorname{Tch}^{\tau}\left(\nabla_{0}^{V}, \nabla_{1}^{V}, \rho\right) \operatorname{ch}^{\tau}\left(\pi_{+}\left(\sigma\left(\mathscr{D}_{E}\right)\right)\right) \operatorname{Todd}_{\tau}(M) d \mu
\end{aligned}
$$

as claimed.

Remark. From Lemma 1.12 we know that the leading symbol of the boundary. Dirac operator $\mathscr{D}^{b}$ and the leading symbol of the Dirac operator $\mathscr{D}$ satisfy $\sigma\left(\mathscr{D}^{b}\right)=c\left(\omega_{p}\right) \sigma(\mathscr{D})$. Since $p$ is odd, $\omega_{p}$ is central in $\operatorname{Cliff}\left(T^{*} \mathscr{F}\right)$ and it is easily seen that $\pi_{+}\left(\sigma\left(\mathscr{D}_{E}^{b}\right)\right)=\pi_{+}\left(\sigma\left(\mathscr{D}_{E}\right)\right)$. Thus formula (2.19) gives the index of the boundary Dirac $\mathscr{D}^{b}$ operator as well.

Next we derive the criteria for the invertibility of Dirac operators

$$
\mathscr{D}_{u}: \Gamma_{\tau}^{\infty}(S \otimes E \otimes V) \rightarrow \Gamma_{\tau}^{\infty}(S \otimes E \otimes V)
$$

If no confusion can arise, the parameter $u$ will be omitted and, if not otherwise stated, all quantities under consideration will be assumed as restricted to some fixed leaf $l$.

Each leaf $l$ of $\mathscr{F}$ is a complete manifold and the Bochner-Weitzenbock identity

$$
\mathscr{D}^{2}=\nabla^{*} \nabla+\frac{1}{4} \kappa+\mathscr{R}_{0}
$$

holds on every $l$. Here $\nabla^{*} \nabla$ is a certain selfadjoint operator, and on simple vectors $\mathscr{R}_{0}$ is given by

$$
\mathscr{R}_{0}(\sigma \otimes e)=\frac{1}{2} \sum_{j, k=1}^{p} e_{j} e_{k} \sigma \otimes R_{e_{j}, e_{k}}^{V \otimes E}(e)
$$


(cf. [GL]). $\kappa$ is the scalar curvature of $l$ and $R_{e_{j}, e_{k}}^{V \otimes E}$ is the curvature transformation of $V \otimes E$ associated to the tensor product connection $\nabla_{u}^{V} \otimes \nabla^{E}$, $0 \leq u \leq 1$. Put $\left\|\mathscr{R}_{0}\right\|=\sup \left\{\left(\mathscr{R}_{0} \varphi, \varphi\right): \varphi \in \Gamma_{\tau}(M, V \otimes E),\|\varphi\|=1\right\}$. Note that there exists a constant $c_{p} \geq 0$ depending only on $p=\operatorname{dim} l$ such that

$$
\left\|\mathscr{R}_{0}^{V \otimes E}\right\| \leq c_{p}\left\|R^{V \otimes E}\right\|
$$

where $R^{V \otimes E}$ is the curvature of $V \otimes E$. The constant $c_{p}$ can be estimated explicitly. It is known that $\kappa \geq 4 \mathscr{R}_{0}^{V \otimes E}$ implies $\operatorname{Ker} \mathscr{D}=\{0\}$ and that $\kappa \geq$ $4 \mathscr{R}_{0}^{V \otimes E}+\varepsilon$ for some $\varepsilon>0$ implies the invertibility of $\mathscr{D}: W_{2}^{1} \rightarrow L^{2}$ (see [GL]). Since the curvature tensor of $V \otimes E$ is a derivation and the curvature 2-form $\Omega_{u}$ of $R^{V}$ is given by $\Omega_{u}=\left(u-u^{2}\right) \psi \wedge \psi$, where $\psi=\nabla^{1}-\nabla^{0} \in \Gamma(\stackrel{*}{\wedge} V \otimes \operatorname{End} V)$, we have

$$
\begin{aligned}
\left\|R^{V \otimes E}\right\| & \leq\left\|R^{V}\right\|+\left\|R^{E}\right\| \leq \max _{0 \leq u \leq 1}\left(u-u^{2}\right)\left\|\psi^{2}\right\|+\left\|R^{E}\right\| \\
& \leq \frac{1}{2}\left\|\psi^{2}\right\|+\left\|R^{E}\right\| .
\end{aligned}
$$

The condition for the invertibility of the family

$$
\mathscr{D}_{u}: \Gamma_{\tau}^{\infty}(S \otimes V \otimes E) \rightarrow \Gamma_{\tau}^{\infty}(S \otimes V \otimes E)
$$

is

$$
\kappa_{l} \geq 4 c_{p}\left(\frac{1}{2}\left\|\psi^{2}\right\|_{l}+\left\|R^{E}\right\|_{l}\right)+\varepsilon, \quad \psi=\nabla^{1}-\nabla^{0}, \varepsilon>0 .
$$

We distinguish two important cases: $E=\varepsilon^{1}$, a one-dimensional trivial bundle, and $E=S$, the spinor bundle over $\mathscr{F}$.

Case 1. $E=\varepsilon^{1}$, then $\mathscr{R}_{0} \equiv 0$ and the condition for the invertibility of the family $\mathscr{D}_{u}: W_{2}^{1} \rightarrow L^{2}$ is

$$
\kappa>2 c_{p}\left\|\nabla^{1}-\nabla^{0}\right\|+\varepsilon \quad(\nu \text {-a.e. }) .
$$

Again by Lemma 1.12 we have that $(2.26)$ is also the condition for the invertibility of the family associated to the boundary Dirac operator.

Case 2. $E=S, R^{s}$ can be estimated in terms of the curvature of the leaf $l$ as follows:

$$
\begin{gathered}
R_{e_{i}, e_{j}}^{S} \sigma=\frac{1}{2} \sum_{p<q}\left\langle R_{e_{i}, e_{j}}\left(e_{p}\right), e_{q}\right\rangle e_{p} e_{q} \sigma . \\
\left\|R^{S}\right\|_{x}^{2}=\sum_{i<j}\left\|R_{e_{i}, e_{j}}^{S}\right\|_{x}^{2} .
\end{gathered}
$$

Now

$$
\begin{aligned}
& \left\|R_{e_{i}, e_{j}}^{S}\right\|^{2}=\sup _{|\sigma|=1}\left\langle R_{e_{i}, e_{j}}^{S} \sigma, R_{e_{i}, e_{j}}^{S} \sigma\right\rangle \\
& \quad=\frac{1}{4} \sup _{|\sigma|=1}\left\langle\sum_{p<q}\left\langle R_{e_{i}, e_{j}} e_{p}, e_{q}\right\rangle e_{p} e_{q} \sigma, \sum_{r<s}\left\langle R_{e_{i}, e_{j}} e_{r}, e_{s}\right\rangle e_{r} e_{s} \sigma\right\rangle \\
& \quad=\frac{1}{4} \sup _{|\sigma|=1} \sum_{p<q} \sum_{r<s}\left\langle R_{e_{i}, e_{j}} e_{p}, e_{q}\right\rangle\left\langle R_{e_{i}, e_{j}} e_{r}, e_{s}\right\rangle\left\langle e_{p} e_{q} \sigma, e_{r} e_{s} \sigma\right\rangle .
\end{aligned}
$$

It is easy to see that only $p=r$ and $q=s$ terms survive, so we get 


$$
=\frac{1}{4} \sum_{p<q}\left|\left\langle R_{e_{i}, e_{j}} e_{p}, e_{q}\right\rangle\right|^{2} \text {. }
$$

Consequently,

$$
\left\|R^{S}\right\|_{x}^{2}=\sum_{i<j}\left\|R_{e_{i}, e_{j}}^{S}\right\|_{x}^{2}=\frac{1}{4} \sum_{i<j} \sum_{p<q}\left|\left\langle R_{e_{i}, e_{j}} e_{p}, e_{q}\right\rangle\right|^{2}=\frac{1}{4}\|R\|_{x}^{2} .
$$

Hence, the condition for the invertibility of the family is

$$
\begin{aligned}
\kappa \geq 2 c_{p}\left(\left\|\psi^{2}\right\|+\frac{1}{2}\|R\|\right)+\varepsilon, \\
\quad \varepsilon>0, R=\text { curv. tensor of } T \mathscr{F} \text { restricted to a leaf } l .
\end{aligned}
$$

To summarize, we have the following corollaries:

Corollary 2.5. Let $\mathscr{D}_{u}: \Gamma_{\tau}^{\infty}(S \otimes V) \rightarrow \Gamma_{\tau}^{\infty}(S \otimes V), 0 \leq u \leq 1$, be a family of operators associated to a Dirac (resp. boundary Dirac) operator via unitary representations $\rho_{i}: \pi_{i}(M) \rightarrow U(n) \quad(i=1,2)$ and an isomorphism $\varphi$. If the metric $g$ on $M$ satisfies the property (2.26) then the eta invariant of the family $\mathscr{D}_{u}$ (resp. $\left.\mathscr{D}_{u}^{b}\right)$ is a smooth real-valued function of the parameter $u$. The local quantity $a_{p}\left(x, \dot{D}_{u}, \mathscr{D}_{u}^{2}\right)$ is computable in terms of the metric and connection jets. As a class in $\bar{H}_{\tau}^{p}(M, \mathbf{R})$, it is given by the formula

$$
a_{p}\left(x, \dot{\mathscr{D}}_{u}, \mathscr{D}_{u}^{2}\right)=\sum_{4 i+2 j-1=p}\left(\frac{\sqrt{-1}}{2 \pi}\right)^{i} \frac{1}{(i-1) !} \widehat{A}_{\tau, j} \cap \operatorname{tr}\left(\psi \cap \Omega_{V}^{i-1}\right)
$$

where $\widehat{A_{\tau}}$ is the tangential A-roof polynomial, $g=g(u)$, and $\psi=\nabla^{1}-\nabla^{0}$.

Corollary 2.6. Let $\mathscr{D}_{u}^{\text {sign }}: \Gamma_{\tau}^{\infty}\left(\wedge^{*} T \mathscr{F} \otimes V\right) \rightarrow \Gamma_{\tau}^{\infty}\left(\wedge^{*} T \mathscr{F} \otimes V\right)$ be a family of operators associated to a boundary signature operator via unitary representations $\rho_{i}: \pi_{i}(M) \rightarrow U(n)(i=1,2)$ and an isomorphism $\varphi$. If condition (2.34) holds true then the eta invariant of the family $\mathscr{D}_{u}^{\mathrm{sign}}$ is a smooth real-valued function of the parameter $u$. The form $a_{p}\left(x, \mathscr{D}_{u}^{\text {sign }},\left(\mathscr{D}_{u}^{\text {sign }}\right)^{2}\right)$ is locally computable in terms of the metric and connection jets. As a class in $\bar{H}_{\tau}^{p}(M, \mathbf{R})$ it is given by the formula

$$
\begin{aligned}
a_{p}\left(x, \dot{\mathscr{D}}_{u}^{\mathrm{sign}},\left(\mathscr{D}_{u}^{\mathrm{sign}}\right)^{2}\right) \\
\quad=\sum_{4 i+2 j-1=p}\left(\frac{\sqrt{-1}}{2 \pi}\right)^{i} \frac{2^{i}}{(i-1) !} L_{j}(g) \cap \operatorname{tr}\left(\psi \cap \Omega_{V}^{i-1}\right)
\end{aligned}
$$

where $L$ is the tangential Hirzebruch polynomial, $g=g(u)$, and $\psi=\nabla^{1}-\nabla^{0}$. Proof of Corollary 2.6. We assume $\operatorname{dim} \mathscr{F}=p, p=4 l+1$. The boundary tangential signature operator is isomorphic to the tangential Dirac operator with 
coefficients in $S$, the total spinor bundle of $T \mathscr{F}$. Hence

$$
\begin{aligned}
& a_{p}\left(x, \dot{\mathscr{D}}_{u}^{\mathrm{sign}},\left(\mathscr{D}_{u}^{\mathrm{sign}}\right)^{2}\right) \\
& \quad=\sum_{4 j+2 i-1=p}\left(\frac{\sqrt{-1}}{2 \pi}\right)^{i} \frac{1}{(i-1) !} \widehat{A}_{\tau, j} \cap \operatorname{tr}\left(\psi \cap \Omega_{S \otimes V}^{i-1}\right)
\end{aligned}
$$

$$
=\sum_{4 j+2 i-1=p}\left(\frac{\sqrt{-1}}{2 \pi}\right)^{i} \frac{1}{(i-1) !} \widehat{A}_{\tau, j} \cap \sum_{p+q=i-1} \frac{(i-1) !}{p ! q !} \operatorname{tr}\left(\Omega_{S}^{q}\right) \operatorname{tr}\left(\psi \cap \Omega_{V}^{p}\right)
$$

$$
=\sum_{4 j+2 i-1=p} \widehat{A}_{\tau, j} \sum_{q+r=i} \operatorname{ch}_{q}^{\tau}(S)\left(\frac{\sqrt{-1}}{2 \pi}\right)^{r} \frac{1}{(r-1) !} \operatorname{tr}\left(\psi \cap \Omega_{V}^{r-1}\right)
$$

$$
=\left\{\sum_{j, q} \widehat{A}_{\tau, j} \cap \operatorname{ch}_{q}^{\tau}(S) \sum_{r}\left(\frac{\sqrt{-1}}{2 \pi}\right)^{r} \frac{1}{(r-1) !} \operatorname{tr}\left(\psi \cap \Omega_{V}^{r-1}\right)\right\}_{p} .
$$

Now, the generating functions for $\mathscr{L}, \widehat{A}$, and $L$ polynomials are

$$
\prod \frac{x_{i} / 2}{\tanh \left(x_{i} / 2\right)}, \quad \prod \frac{x_{i}}{\exp \left(x_{i} / 2\right)-\exp \left(-x_{i} / 2\right)}, \quad \prod \frac{x_{i}}{\tanh x_{i}}
$$

respectively. Also, $\operatorname{ch}_{q}(S)=\prod\left(\exp \left(x_{i} / 2\right)+\exp \left(-x_{i} / 2\right)\right), \operatorname{ch}_{q}(S)=0, q$ odd (cf. [APS]). Thus we have $2^{p+1} \mathscr{L}=\operatorname{ch}(S) \widehat{A}$ and $\mathscr{L}_{s}=2^{-2 s} L_{s}$, i.e., $\widehat{A}_{j} \operatorname{ch}_{q}(S)=$ $2^{(p+1-4 s) / 2} L_{s}, 4 s=4 j+2 q$. Applying the "restriction" homomorphism

$$
r_{*}: H_{D R}^{*}(M, \mathbf{R}) \rightarrow \bar{H}_{\tau}^{*}(M, \mathbf{R})
$$

gives the equality for tangential characteristic classes. Substituting it in (2.40) gives the claimed result, i.e., (2.36).

Remark. Connes and Skandalis construct in [CS] a map

$$
\operatorname{Ind}_{t}^{*}: K_{\xi}^{*}(B G(M, \mathscr{F})) \rightarrow K_{*}\left(C_{r}^{*}(M, \mathscr{F})\right)
$$

from the $\xi$-twisted $K$-theory of $B G(M, \mathscr{F})$ to the $C^{*}$-algebraic $K$-theory of $C_{r}^{*}(M, \mathscr{F})$, which they call the topological index map. The relative index map $\operatorname{ind}_{\nu}^{1}\left(\rho_{0}, \rho_{1}, \varphi, D\right)$ factors through $K^{0}(S(M, \mathscr{F}))$ (cf. [P1]). Also, there is a natural map of $K$-theories, $\delta: K^{0}(S(M, \mathscr{F})) \rightarrow K_{\xi}^{1}(B G(M, \mathscr{F}))$, obtained from the exact sequence of the pair $(B(\xi), S(\xi))$. Thus there is a well-defined map

$$
\operatorname{Ind}_{t}^{1} \circ \delta: K^{0}(S(M, \mathscr{F})) \rightarrow K_{\xi}^{1}(B G(M, \mathscr{F})) \rightarrow K_{1}\left(C_{r}^{*}(M, \mathscr{F})\right)
$$

and therefore a well-defined element $\operatorname{Ind}_{t}^{1} \circ \delta\left[\sigma_{D}\right] \in K_{1}\left(C_{r}^{*}(M, \mathscr{F})\right)$, where $\left[\sigma_{D}\right] \in K^{0}(S(M, \mathscr{F}))$ is the symbol class of a tangential pseudodifferential operator $D$.

The generalization of Theorems 2.1 and 2.2 would be to identify $\operatorname{Ind}_{t}^{1} \circ \delta\left[\sigma_{D}\right]$ and to show that if $\mathscr{F}$ admits a holonomy invariant measure $\nu$ then

(2.43) $c \operatorname{Ind}_{t}^{1} \delta\left[\sigma_{D}\right]=\sum_{4 i+2 j=p-1}\left(\frac{\sqrt{-1}}{2 \pi}\right)^{j} \frac{1}{(j-1) !} \widehat{A}_{\tau, i} \cap \operatorname{tr}\left(\psi \cap \Omega_{E}^{j-1}\right) \in \bar{H}_{\tau}^{p}(M)$ 
and

$$
\begin{aligned}
\Phi_{\nu} \operatorname{Ind}_{t}^{1} \delta\left[\sigma_{D}\right] & =\operatorname{ind}_{\nu}^{1}\left(\rho_{0}, \rho_{1}, \varphi, D\right) \\
& =\left\langle\operatorname{Tch}_{\tau}\left(\nabla_{0}, \nabla_{1}\right) \operatorname{ch}_{\tau}\left(\pi_{+}(\sigma(D)) \operatorname{Todd}_{\tau}(M),[C]\right\rangle\right.
\end{aligned}
$$

Here $c: K_{1}\left(C_{r}^{*}(M, \mathscr{F})\right) \rightarrow \bar{H}_{\tau}^{p}(M)$ is the suspension of a partial Chern character (cf. [MS, H]). This will be taken up in [P3].

\section{INDEX THEOREM ON A FOLIATED CYLINDER}

Assume that $(M, \mathscr{F})$ is a compact foliated spin manifold, $\operatorname{dim} \mathscr{F}$ odd, and $\nu$ a holonomy invariant transverse measure on $\mathscr{F}$. On the foliated cylinder $(M \times[0,1], \mathscr{F} \times[0,1])$ construct the holonomy invariant measure $\mu=\nu \times \lambda$, where $\lambda$ is the Lebesgue measure on $[0,1]$.

Let $\mathscr{D}_{E}$ be a tangential Dirac operator $\mathscr{D}_{E}: \Gamma_{\tau}^{\infty}(M, S \otimes E) \rightarrow \Gamma_{\tau}^{\infty}(M, S \otimes E)$ with coefficients in a bundle $E \rightarrow M$. $\mathscr{D}_{E}$ can be identified as the restriction to the boundary of $\mathscr{D}_{E}^{+}: \Gamma_{\tau}^{\infty}\left(M \times[0,1], S^{+} \otimes E\right) \rightarrow \Gamma_{\tau}^{\infty}\left(M \times[0,1], S^{-} \otimes E\right)$, where $S^{+}$is the half spinor bundle over $M \times[0,1],\left.S^{+}\right|_{M} \approx S$.

In order to have a well-defined boundary problem assume that $\mathscr{D}$ restricted to the leaves of $M$ is invertible (see [BC] for the corresponding discussion on fibrations). Topologically, invertibility means that $\mathscr{D}$ is trivial in

$$
K K^{1}\left(C(M), C_{r}^{*}(M, \mathscr{F})\right) .
$$

Impose the global Atiyah-Patodi-Singer boundary conditions. Then there is a strong evidence that the following is true:

Conjecture. $\mathscr{D}_{E}^{+}: \Gamma_{\tau}^{\infty}\left(M \times[0,1], S^{+} \otimes E\right) \rightarrow \Gamma_{\tau}^{\infty}\left(M \times[0,1], S^{-} \otimes E\right)$ is a Fredholm operator in the sense of Breuer in $W^{*}(M \times[0,1], \mathscr{F} \times[0,1])$ and its index is given by the (expected) formula

$$
\operatorname{index}_{\mu}\left(\mathscr{D}_{E}^{+}\right)=\left\langle\widehat{A}_{\tau} \operatorname{ch}_{\tau}(E),\left[C_{\nu}\right]\right\rangle+\frac{1}{2} \eta(0) .
$$

Example 1. Let $(M, \mathscr{F})$ be a compact foliated manifold, $\nu$ an invariant transverse measure, $\operatorname{dim} \mathscr{F}$ odd. Assume $g_{i}(i=1,2)$ are the two metrics on $T \mathscr{F}$ so that $g_{i}$ lies in the same connected component of the space of metrics of $(\nu$ a.e.) positive scalar curvature. Let $\gamma(u)(0 \leq u \leq 1)$ be a curve joining $g_{1}$ and $g_{2}$.

Consider the family $\mathscr{D}_{u}$ of Dirac operators associated to a metric $g_{\gamma(u)}$ and a corresponding eta invariant $\eta\left(\mathscr{D}_{u}\right)$. Then,

$$
\eta\left(g_{2}\right)-\eta\left(g_{1}\right)=\int_{M \times I} \widehat{A_{\tau}}(x, t) d(\nu \otimes \lambda)
$$

where $d(\nu \otimes \lambda)$ is the holonomy invariant measure for the foliation $\mathscr{F} \times I$ obtained from $\nu$ and the standard Lebesgue measure $\lambda$ on the unit interval $I$.

Example 2. Suppose $M$ is foliated by $(\nu$ a.e.) compact leaves, $\operatorname{dim} \mathscr{F}=$ $4 k-1$. Pick any two metrics $g_{i}(i=1,2)$ and a path $\gamma$ joining them. Construct a family of boundary signature operators $\mathscr{D}_{u}^{\text {sign }}$ associated to the given metrics.

Although this family is not invertible it has the property that on every leaf $l, \operatorname{dim} \operatorname{Ker} \mathscr{D}_{u}^{\text {sign }}=$ const . (equal to the appropriate Betti number), and thus 
$\eta\left(\mathscr{D}_{u}^{\text {sign }}\right)$ is a well-defined R-valued differentiable function (cf. [APS]).

$$
\eta\left(g_{2}\right)-\eta\left(g_{1}\right)=\int_{M} L_{\tau}(x, t) d(\nu \otimes \lambda) .
$$

Remark. Example 2 can be generalized to foliations obtained by suspension, i.e. foliations on $M=\widetilde{X} \times_{\pi_{1}(X)} U(n)$, where $\widetilde{X}$ is the universal covering of some compact manifold $X$ and $\pi_{1}(X)$ acts on the unitary group $U(n)$. The leaf of $M$ passing through $[x$, id $] \in \widetilde{X}_{\pi_{1}(X)} \times U(n)$ is the covering space $\tilde{X} /\left\{g \in \pi_{1}(X), g x=x\right\}$ of $X, x \in X$.

Let us now discuss the homotopy invariance of the variation $\eta_{\alpha_{1}}(0)-\eta_{\alpha_{2}}(0)$ in the presence of the conjecture.

Denote by $R$ the space of all unitary $n$-dimensional representations of the fundamental group $\pi_{1}(M) \equiv \pi$ of the compact foliated space $M$. Let $\alpha_{1}, \alpha_{2}$ be elements of $R$ and $\gamma$ a curve in $R$ joining $\alpha_{1}$ and $\alpha_{2}$. Let $E \pi \rightarrow B \pi$ be a principal $\pi$ bundle with contractible total space $E \pi$. Then $B \pi$ is called the classifying space of the group $\pi$. Let $f: M \rightarrow B \pi$ be a map classifying the universal cover $\widetilde{M}$ of $M$.

Construct a rank $n$ vector bundle $F \rightarrow B \pi \times R$ over $B \pi \times R$ as follows: consider the action of $\pi$ on $E \pi \times R \times C^{n}$ given by

$$
\begin{gathered}
\left(E \pi \times R \times \mathbf{C}^{n}\right) \times \pi \rightarrow E \pi \times R \times \mathbf{C}^{n}, \\
((e, \alpha, v), \gamma) \rightarrow(e \gamma, \alpha, \alpha(\gamma) v) .
\end{gathered}
$$

Define the vector bundle $F$ over $B \pi \times R$ to be $\left(E \pi \times R \times C^{n}\right) / \pi$. The property of $F$ is that $\left.F\right|_{B \pi \times \alpha}$ is the flat unitary vector bundle over $B \pi$ defined by $\alpha$. Let $E=(f \times 1)^{*} F$ be the vector bundle over $M \times R$. By the Künneth Theorem in cohomology, we have $\operatorname{ch}(F)=\sum_{i} x_{i} \xi_{i}, x_{i} \in H^{*}(B \pi, \mathbf{R}), \xi_{i} \in H^{*}(R, \mathbf{R})$. It follows that if $y_{i}=f^{*}\left(x_{i}\right)$, then $\operatorname{ch}(E)=\sum_{i} y_{i} \xi_{i}$.

We consider $B \pi$ and $R$ as spaces foliated by one leaf so that $\operatorname{ch}(F)$ and $\operatorname{ch}(E)$ can be interpreted as tangential cohomology classes.

Proposition 3.1. Let $(M, \mathscr{F}, S)$ be a compact foliated spin manifold. Assume that $M$ admits a Riemannian metric with leafwise positive scalar curvature. Then, as in the notation above,

$$
\eta_{\alpha_{1}}(0)-\eta_{\alpha_{2}}(0)=\sum_{i} \int_{M} \widehat{A}_{\tau}(M) y_{i} d \nu \int_{\gamma} \xi_{i} d \lambda .
$$

Proof. Let $S^{+} \rightarrow M \times \gamma$ be a spin bundle over $M \times \gamma$ associated to the irreducible representation of $\operatorname{Spin}(\operatorname{dim} \mathscr{F}+1)$, and let $\mathscr{D}_{E}^{+}$be the associated Dirac operator, defined using the metric $g \otimes d t^{2}$ and acting on the space of tangentially smooth section of $\left.S^{+} \otimes E\right|_{M \times \gamma}$. If $g$ has a positive scalar curvature on $M$ so does $g \otimes d t^{2}$ on $M \times \gamma$. By a standard argument using the Weitzenböck formula and the fact that $\left.E\right|_{M \times \gamma}$ is flat, we conclude that $\operatorname{ind}_{\Lambda} \mathscr{D}_{E}^{+}$vanishes. According to the conjecture,

$$
\begin{aligned}
0 & =\operatorname{ind}_{\mu}\left(\mathscr{D}_{E}^{+}\right) \\
& =\int_{M \times \gamma} \widehat{A}_{\tau}(M \times \gamma) \operatorname{ch}(E) d(\nu \otimes \lambda)+\frac{1}{2}\left(\eta_{\alpha_{1}}(0)-\eta_{\alpha_{2}}(0)\right) .
\end{aligned}
$$


From the discussion above,

$$
\int_{M \times \gamma} \widehat{A}_{\tau}(M \times \gamma) \operatorname{ch}(E) d(\nu \otimes \lambda)=\sum_{i} \int_{M} \widehat{A}_{\tau}(M) y_{i} d \nu \int_{\gamma} \xi_{i} d \lambda
$$

(using multiplicativity of $\widehat{A_{\tau}}$ polynomial). This completes the proof.

Corollary 3.2. Suppose that the assumptions of Proposition 3.1 are satisfied. Then the variation $\eta_{\alpha_{1}}(0)-\eta_{\alpha_{2}}(0)$ is homotopy invariant with respect to $\alpha_{i}$ $(i=1,2)$ if

$$
\sum_{i} \int_{M} \widehat{A}_{\tau}(M) y_{i} d \nu=0 .
$$

Corollary (3.2) is closely related to the generalized J. Rosenberg's conjecture: If $(M, \mathscr{F})$ is a spin foliation of a closed manifold $M$ admitting a metric of leafwise positive scalar curvature, and if $\pi \cong \pi_{1}(M)$, then for all $x \in$ $H^{*}(B \pi, \mathbf{R})$,

$$
\int_{M} \widehat{A}_{\tau} f^{*}(x)=0
$$

\section{APPENDIX}

We prove the statement needed in Theorem 2.1, that is, if $\omega$ is a tangentially smooth tangential form such that

$$
\left.\frac{d \eta\left(d_{u}, s\right)}{d u}\right|_{s=0}=\int \omega d \nu
$$

where the family $\mathscr{D}_{u}$ of Dirac operators is as described in Theorem 2.1, then the restriction $\left.\omega\right|_{l}$ of $\omega$ to a leaf $l$ can be evaluated according to the rule

$$
\left.\omega\right|_{l}=\sum_{4 i+2 j=p-1}\left(\frac{\sqrt{-1}}{2 \pi}\right)^{j} \frac{1}{(j-1) !} \widehat{A}_{\tau, i} \cap \operatorname{tr}\left(\psi \cap \Omega_{E \otimes V}^{j-1}\right),
$$

$\left.\omega\right|_{l}$ locally computable (cf. Proposition 1.11), and according to Gilkey's theory is expressible in terms of the universal characteristic forms, depending on the dimension but not on the manifold.

Proposition 4.1. Let $\mathscr{D}_{u}: \Gamma_{\tau}^{\infty}(M, \mathscr{F}, S \otimes E) \rightarrow \Gamma_{\tau}^{\infty}(M, \mathscr{F}, S \otimes E)$ be an invertible family of tangential Dirac operators depending smoothly on $u \in[0,1]$. Then the restriction of the form $\omega$ as above satisfies

$$
\left.\omega\right|_{l}=\sum_{4 i+2 j=p-1}\left(\frac{\sqrt{-1}}{2 \pi}\right)^{j} \frac{1}{(j-1) !} \widehat{A}_{\tau, i} \cap \operatorname{tr}\left(\psi \cap \Omega_{E}^{j-1}\right) .
$$

Similar computations are done in several places so we will be brief. According to analysis as in [G1], it can be shown that $\omega=\left.\omega\right|_{l}$ has the general form

$$
\omega=\sum_{4 i+2 j=p-1} P_{i}(g) \cap \operatorname{tr}\left(\psi \cap \Omega_{E}^{j-1}\right)
$$

where $P_{i}(g)$ is the characteristic $4 i$-form of $T l$. We evaluate $P_{i}(g)$ using compact spin $l$ 's. The following lemma is well known. 
Lemma 4.2. Let $M_{i}(i=1,2)$ be oriented compact and spin Riemannian manifolds, and let $W_{i}$ be coefficient bundles over $M_{i}$ with given connections $\nabla_{r}$. Let $M=M_{1} \times M_{2}$ and $W_{1} \otimes W_{2}$ with the product metric and connection. Let $\operatorname{dim} M_{1}$ be odd and $\operatorname{dim} M_{2}$ be even. Let $\mathscr{D}_{1}$ (resp. $\left.\mathscr{D}_{2}\right)$ be the boundary Dirac operators over $M$ (resp. $\left.M_{1}\right)$ with coefficients in $W$ (resp. $\left.W_{1}\right)$. Let $\mathscr{D}$ be the (half) Dirac operator over $M_{2}$ with coefficients in $W_{2}$. Then $\eta\left(\mathscr{D}_{1}\right)=\eta\left(\mathscr{D}_{2}\right)$ index $(\mathscr{D})$.

Proof of Proposition 4.1. Choose $i, 0 \leq i \leq p / 4$, and let $4 i+2 j-1=p$. Let $\operatorname{dim} M_{1}=2 j-1, \operatorname{dim} M_{2}=4 i$. Assume that the Pontriagin classes of $M_{1}$ are trivial (this can be easily done). Let $\left(W, \nabla_{W}\right)$ be the pullback of a bundle and connection over $M_{1}$. Then

$$
\begin{aligned}
\frac{d \eta\left(\mathscr{D}_{1}\right)}{d u} & =\int_{M_{2}} P_{i}(g)_{M_{2}} \int_{M_{1}} \operatorname{tr}\left(\psi \cap \Omega_{W}^{j-1}\right) \\
& =\int_{M_{2}} P_{0}(g)_{M_{2}} \int_{M_{1}} \operatorname{tr}\left(\psi \cap \Omega_{W}^{j-1}\right)
\end{aligned}
$$

by (4.4). By Lemma 4.2,

$$
\begin{aligned}
\frac{d \eta\left(\mathscr{D}_{1}\right)}{d u} & =\frac{d \eta\left(\mathscr{D}_{2}\right) \text { index }(\mathscr{D})}{d u} \\
& =\int_{M_{1}} P_{0}(g)_{M_{2}} \operatorname{tr}\left(\psi \cap \Omega_{W}^{j-1}\right) \int_{M_{2}} \widehat{A}_{i}(g) .
\end{aligned}
$$

Since $d \eta\left(\mathscr{D}_{2}\right) / d u$ is not zero it follows that

$$
\int_{M_{2}} P_{0}(g)_{M_{2}} \widehat{A_{i}}(g)=\int_{M_{2}} P_{i}(g) \text { for every } M_{2} .
$$

From the well-known fact that the equality of the integrals yields the equality of the characteristic forms, we conclude that $P_{i}(g)$ is the scalar multiple of the $A$-roof genus,

$$
P_{0}(g) \widehat{A_{i}}=P_{i}(g)
$$

or

$$
\left.\omega\right|_{l}=\sum_{4 i+2 j=p-1} c(j) \widehat{A}_{i}(g) \cap \operatorname{tr}\left(\psi \cap \Omega_{W}^{j-1}\right) .
$$

To determine the scalar $c(v)$ let $M_{1}=S^{1}$, let $W_{1}$ be a trivial bundle over $S^{1}$, and let $\psi^{1}$ be any form with $\int \psi^{1} \neq 0$. Let $\nabla(u)$ have connection 1form $u \psi, M_{2}=T^{p-1}$ a flat torus, and let $W_{2}$ be a bundle over $M_{2}$ so that $\int_{M_{2}} \operatorname{ch}_{k-1}\left(W_{2}\right) \neq 0, k=(p-1) / 2$. Let $M=M_{1} \times M_{2}$ and $\dot{W}=W_{1} \otimes W_{2}$. Then

$$
\frac{d \eta\left(\mathscr{D}_{1}\right)}{d u}=\int_{M} c(k) \operatorname{tr}\left(\psi \cap \Omega_{W}^{k-1}\right)=\int_{S^{1}} c(k) \psi \int_{T^{p-1}} \operatorname{tr}\left(\Omega_{W}^{k-1}\right) .
$$

On the other hand,

$$
\begin{aligned}
\frac{d \eta\left(\mathscr{D}_{1}\right)}{d u} & =\frac{d \eta\left(\mathscr{D}_{2}\right)}{d u} \operatorname{index}(\mathscr{D}) \\
& =\int_{S^{1}} c(1) \psi \int_{T^{p-1}}\left(\frac{\sqrt{-1}}{2 \pi}\right)^{k-1} \frac{1}{(k-1) !} \operatorname{tr}\left(\Omega_{W}^{k-1}\right)
\end{aligned}
$$


Comparing the two last equations, we get

$$
c(k)=c(1)\left(\frac{\sqrt{-1}}{2 \pi}\right)^{k-1} \frac{1}{(k-1) !} .
$$

That $c(1)=\sqrt{-1} / 2 \pi$ can be checked directly using the family of Dirac operators

$$
\mathscr{D}_{u}=\frac{1}{\sqrt{-1}} \frac{d}{d \theta}-u
$$

on the unit circle $S^{1}$. This completes the proof of Proposition 4.1.

\section{BIBLIOGRAPHY}

[ABP] M. F. Atiyah, R. Bott, and V. K. Patodi, On the heat equation and index theorem, Invent. Math. 19 (1973), 279-330.

[APS] M. F. Atiyah, V. K. Patodi, and I. M. Singer, Spectral asymmetry and Riemannian geometry. I, Math. Proc. Cambridge Philos. Soc. 77 (1975), 43-69; II, 78 (1975), 405-432; III, 79 (1976), 71-99.

[BC] J. M. Bismut and J. Cheeger, Families index for manifolds with boundary, superconnections and cones, Preprint.

[BF] J. M. Bismut and D. S. Fried, The analysis of elliptic families, Comm. Math. Phys. 107 (1986), 103-163.

[Bt] L. Boutet de Montvel, A course on pseudodifferential operators and their applications, Duke Univ. Math. Ser. II (to appear).

[CG] J. Cheeger and M. Gromov, On characteristic numbers of complete manifolds of bounded curvature and finite volume, Rauch Memorial Volume, Springer-Verlag, 1985, pp. 115-154.

[C1] A. Connes, Sur la théorie non commutative de l'intégration, Lecture Notes in Math., Vol. 725, Springer-Verlag, 1979, pp. 19-143.

[C2] - A survey of foliations and operator algebras, Operator Algebras and Applications, Proc. Sympos. Pure Math., Vol. 38, Amer. Math. Soc., Providence, R.I., 1980.

[CS] A. Connes and G. Skandalis, The longitudinal index theorem for foliations, Publ. Res. Inst. Math. Sci. 20 (1984), 1139-1183.

[DHK] R. G. Douglas, S. Hurder, and J. Kaminker, Cyclic cocycles, renormalization and eta invariants, Preprint, 1990.

[E] M. A. Evgrafov, Analytic functions, Saunders, 1966.

[G1] P. Gilkey, Invariance theory, the heat equation, and the Atiyah-Singer index theorem, Publish or Perish, 1984.

[G2] _ The eta invariant and secondary characteristic classes of locally flat bundles, TeubnerTexte Math. 70 (1984).

[GL] M. Gromov and B. Lawson, Positive scalar curvature and the Dirac operator on Riemannian complete manifolds, Publ. Math. Inst. Hautes Études Sci. 58 (1983), 295-408.

[H] S. Hurder, The $\bar{\partial}$ operator, Global Analysis on Foliated Spaces, Appendix A, MSRI Publications, 1988.

[LM] H. B. Lawson, Jr. and M. L. Michelsohn, Spin geometry, Princeton Univ. Press, Princeton, N.J., 1989.

[MS] C. C. Moore and C. Schochet, Global analysis on foliated spaces, MSRI Publications, 1988.

[MoS] H. Moscovici and R. Stanton, Eta invariants of Dirac operators on locally symmetric spaces, Invent. Math. 95 (1989), 626-666.

[P1] G. Perić, Ph.D. Thesis, The Ohio State University, 1989.

[P2] _ Index theorem for foliated manifolds with boundary and cyclic cocycles, Preprint, 1990.

[P3] _ Type III relative index theorem on foliated manifolds, in preparation, 1990. 
[R] J. Roe, An index theorem on open manifolds. I, J. Differential Geom. 27 (1988), 87-113.

[RS] D. Ruelle and D. Sullivan, Currents, flows and diffeomorphisms, Topology 114 (1975), 319327.

[S] R. Seeley, Complex powers of an elliptic operator, Proc. Sympos. Pure Math., Vol. 10, Amer. Math. Soc., Providence, R.I., 1967, pp. 288-307.

[Sh] P. Shanahan, The Atiyah-Singer index theorem, Lecture Notes in Math., Vol. 693, SpringerVerlag, 1978.

[V] V. Mathai, Positive scalar curvature and reduced eta invariants, Preprint.

[Y] K. Yoshida, Functional analysis, Springer-Verlag, 1965.

Department of Mathematics, The Ohio State University, Columbus, Ohio 43210

Current address: Institut für Augsburg, 9800 Augsburg, Germany

E-mail address: Bruening@uni-augsburg.de 